\title{
Parental Social Support and Adolescent Well-Being : a Cross-Sectional Study in China
}

\author{
Wang, Ziyu
}

2019-02

Wang , Z , Kouvonen , A M , Satka, M E A \& Julkunen , I H G 2019 , ' Parental Social Support and Adolescent Well-Being : a Cross-Sectional Study in China ', Child Indicators pÿResearch , vol. 12 , no. 1 , pp. 299317 . https://doi.org/10.1007/s12187-018-9547-2

http://hdl.handle.net/10138/300150

https://doi.org/10.1007/s12187-018-9547-2

acceptedVersion

Downloaded from Helda, University of Helsinki institutional repository.

This is an electronic reprint of the original article.

This reprint may differ from the original in pagination and typographic detail.

Please cite the original version. 


\title{
Parental social support and adolescent well-being: a cross-sectional study in China
}

\begin{abstract}
The aim of this study was to examine whether the two components of parental social support, emotional and instrumental, are associated with various aspects of adolescent well-being in the current Chinese context. A sample of 1306 adolescents (47\% girls, 11, 13 and 15 years old, 39\% urban) was derived from the nationally representative 2012 survey "China Family Panel Studies". Four indicators of adolescent well-being were examined: health status, academic attainment, self-perception and depression. Logistic regression and multiple linear regression models were applied to analyze associations between the two domains of parental social support and four indicators of adolescent well-being, respectively. Both the intermediate and high levels of emotional support were associated with better self-perception and lower levels of depression, while these associations were only reflected at the high level of instrumental support. In addition, only the high level of emotional support was associated with higher academic attainment. Overall these associations remained after controlling for socio-demographic characteristics, including age, gender, living location, family income and family size. However, some of the confounders were more influential on adolescent well-being than others. For example, those adolescents who lived in rural areas were more likely to report suboptimal health status. These findings are discussed in the context of the rapid changes in Chinese society. This research adds to the empirical evidence on the association between parental social support and adolescent well-being in contemporary Mainland China.
\end{abstract}

Keywords Adolescent · Child · Social support · Well-being · Parent · China 


\section{Introduction}

There is an increasing international consensus that parental social support is a crucial factor in determining adolescents' wellbeing. In general, parental behaviors are positively associated with optimal outcomes, such as positive self-image (Bean et al. 2003; Mcmurray et al. 2011; Noble-Carr et al. 2013), healthy life experience (Uchino 2006, 2009) and better school performance (Gordon and Cui 2012; Rueger et al. 2010). Continual lack of support from parents, on the contrary, tends to lead to maladaptive outcomes in adolescents, such as substance use (Bond et al. 2007; Wills et al. 2014), smoking (Wills et al. 2017), alcohol use (Kristjansson et al. 2010) and depression (Desjardins and Leadbeater 2011; Stice et al. 2004). Parental social support, especially compared with peer support, is beneficial to the well-being of adolescents. Despite the overall confirmation of the protective function, it is still unknown which aspects of parental social support are critical for positive outcomes in adolescents. Although much literature has discussed the distinct influences of perceived and received social support (see two reviews Chu et al. 2010; Haber et al. 2007), few studies have concentrated on the differences between emotional and instrumental types of support, particular in the context of parental social support.

As an important source of social support for adolescents, parental social support refers to the availability of resources parents can provide their children to help them cope with problems (Wills et al. 2014). Parental social support is usually divided into two functional categories: emotional support and instrumental support (Cutrona and Russell 1987; ShakespeareFinch and Obst 2011; Wills et al. 1992). Generally, emotional support is linked to behavior and attitudes of respect, care, warmth and love, whereas instrumental support refers to practical assistance. Most studies tend to measure total social support without distinguishing between the different aspects of support, that is, emotional and instrumental. Recently, however, an emerging literature has demonstrated the differing effects of emotional and instrumental domains on occupational and interpersonal relationships (Cheung and Sim 2017), self-concept (Zhu et al. 2014) and suicidal ideation (Park et al. 2010) in Asian cultures. Notably, a recent study, examining groups of close friends, discovered that emotional support, instead of instrumental support, consistently accompanies subjective well-being (Morelli et al. 2015).

Since the 1990s, studies of child ${ }^{1}$ well-being have moved beyond the use of a single overall indicator to a more multifaceted understanding, based on life course, child-centered and rights-based theories (Ben-Arieh et al. 2014; Pollard and Lee 2003; Ryan et al. 2008; Ryff 1989; Seligman 2011; Waterman 1993). Despite various concerns in different countries, ongoing considerations of measuring child well-being generally involve the six indicators listed by UNICEF: material well-being, health and safety, educational well-being, relationships, behaviors and risks, and subjective well-being (Adamson 2007; Bradshaw and Richardson 2009; Casas 2011; Martorano et al. 2014). Within such a multifaceted framework, the nominal work "Report on the State of Children in China" produced a full picture of the well-being of Chinese children in 2010 (Chen et al. 2015). This report, however, examined the well-being of the whole age range of children, rather than focusing precisely on adolescents.

A large body of literature has provided theoretical explanations for the association between parental social support and the single factor of well- (or ill-) being, of which the foundational theory is the "stress buffering" function of social support in protecting adolescents from stress and adverse life experience (Cohen and Wills 1985; Cutrona and Russell 1987). Recently,

\footnotetext{
${ }^{1}$ Child, in this article, refers to the UN definition of population aged 0-18 years old, including adolescents.
} 
studies have increasingly concentrated on explaining how social support impacts well-being (Feeney and Collins 2015), physical health (Uchino et al. 2012) and mental health (Lakey and Orehek 2011) in a broader manner.

Although China has the second-largest adolescent population in the world (UNICEF 2012), the relationship between parental support and adolescents' well-being remains a relatively neglected area, especially given the lack of empirical evidence on the broader national situation. Despite the dramatic societal transformation of the past 40 years, traditional collectivism norms still have a strong effect on both adolescents and their parents (Chao and Tseng 2002; Smetana 2011). Nevertheless, due to globalization and the one-child policy enacted in 1979, currently parent-child relationships increasingly challenge the traditional strong bonding and family obligations, and display child-centered, equal and interactive characteristics, similar to those seen in Western countries (Cheng and Berman 2012; Liu 2010). Further study is required to investigate the influence of this change of parenting style on adolescents. In addition, the state has expanded welfare coverage and improved the quality of social services, particularly in the last 10 years, which has led to a general improvement in the well-being of children. The general social welfare reform has alleviated poverty and improved medical care for children since 2003. Compulsory education became free from tuition fees nationally in 2008. The "two exemptions and one subsidy" policy aims to improve access to education and equity for children from poor families. With its rapid societal changes and gradual state intervention, China provides a unique context to investigate the association between parental support and child wellbeing.

The aim of this study is to investigate whether the two components of parental social support, emotional and instrumental, are associated with the different aspects of adolescent well-being in the contemporary Chinese context. By measuring adolescent well-being as a multidimensional construct, we assume that both emotional and instrumental support are associated with the health, academic attainment, self-perception and mental health of Chinese adolescents. To our knowledge, this is the first study to use nationally representative data to analyze the association between parental social support and adolescent wellbeing in Mainland China.

\section{Methods}

\subsection{Data and participants}

China Family Panel Studies (CFPS) is an ongoing nationally representative survey, launched by Peking University, to collect individual-, family-, and community-level longitudinal data in contemporary China, with an 84\% response rate in the 2010 sample (Xie and $\mathrm{Hu}$ 2014). The 2012 sample covered 25 municipalities (13315 families) to represent the social, economic, education and health situation of Chinese families. Of the 8620 child participants ( 0 to 15 years old, $47.5 \%$ girls), 3056 were adolescents from 10 to 15 years old (48\% girls). Since the participants answering parental social support questions were 11, 13 and 15 years old, the current study is was also limited to these age groups $(\mathrm{N}=1541)$. The selected data were mainly reported by adolescents, except for family income and family size, which were reported by their caregivers. The missing values were handled by the listwise deletion method, whereby 235 cases were deleted. All these conditions yielded 1306 cases (11, 13 and 15 years old, 47\% girls) for the analysis in total. The data are openly accessible (Isss 2015).

\subsection{Measures}




\subsubsection{Parental social support}

CFPS measured parental social support with a 14-item inventory. In line with previous literature, all items could be further divided into two dimensions: instrumental support and emotional support. The questions about emotional support were derived from the "care" domain of the highly reliable "Parental Bonding Instrument" (PBI) (Parker et al. 1979; Wilhelm et al. 2005). According to Parker's original design, the "care" domain in PBI reflects support or closeness from parents. Items 1,18 and 24 of PBI were either originally or reversely phrased in CFPS. An example of the items regarding emotional support in CFPS is "Your parents encourage you to do things with great effort". In terms of the subscale of instrumental support, CFPS included assistance with schooling (e,g., "Your parents help you with your schoolwork") and spending time together (e.g., "Your parents play with you - for example, playing chess or playing outdoors"). Responses were scored on a 5-point Likert type scale, ranging from $1=$ "never" to $5=$ "always".

The construct validity was examined through confirmatory factor analysis (CFA). First, exploratory factor analysis (EFA) was conducted to explore the potential factor structures. The initial Kaiser criterion and the screen test suggested two- and three-factor solutions. In line with social support theory, this research selected the two-factor structure, which accounted for $41 \%$ for the variance. A further Promax rotation confirmed this structure with a number of strong loadings greater than 0.30 (Brown 2015). Two items ("Your parents criticize you" and "Your parents attend parent-teacher meetings at school") did not load. Both of them were therefore omitted. As a result, 8 items were used to assess emotional support and 4 items instrumental support. Second, CFA was performed to validate the two-factor construct, using SAS version 9.4. Since the data presented a non-normal distribution, the goodness of fit was evaluated using the robust maximum likelihood (ML) estimation. The model fit indices were as follows: $\chi^{2}=388.77, \mathrm{df}=53, \mathrm{p}<0.001$; Standardized Root Mean Square Residual (SRMR) $=0.06$; Root Mean Square Error of Approximation (RMSEA) $=0.06$ [90\% CI: 0.05-0.07]; Comparative Fit Index (CFI) $=0.91$. Using the recommended cutoffs of $>0.90$ for CFI and $<0.08$ for RMSEA and for SRMR (Hair et al. 2010; Schermelleh-Engel et al. 2003), the two-factor structure was confirmed as an acceptable fit. Factor loadings (standardized) ranged from 0.46 to 0.76. The correlation between the two domains was $\mathrm{r}=0.61(\mathrm{p}<0.001)$.

The final summary scales of the two components manifested high reliability, with Cronbach alpha coefficient of 0.78 for the emotional support domain and alpha of 0.72 for the instrumental. For further analysis, both emotional and instrumental support were categorized into low, intermediate and high groups, with 33\% and 66\% cut-off points (Kouvonen et al. 2012; Stansfeld et al. 2013).

\subsubsection{Health status}

Self-rated health status served as the physical health component of adolescents' well-being. The value of using children's self-reports about their health situation is increasing in clinical pediatric and well-being studies (Varni et al. 2007), especially given the high credibility of this instrument in adolescents (Riley 2004). In CFPS, this variable was worded as "How would you rate your health status?" and the responses were scored on a 5-point Likert Scale from "1=excellent" to "5=poor". In our sample, scores 4 and 5 were categorized as suboptimal health (coded as 1), while scores from 1 to 3 were categorized as good heath (coded as 0 ). Only $7 \%$ of the sample belonged to the suboptimal health group. 


\subsubsection{Academic attainment}

Academic attainment was assessed by a cognitive test. Inspired by the Guttmann Scale design, participants were asked to recognize a series of 34 Chinese characters. Questions were ranked from easy to difficult in order. The participants were asked to memorize 10 Chinese words, and then to recall them after two and four minutes. The final scores were summarized after two rounds of the memorizing process. Since the performance of this test is highly dependent on educational level, starting levels were different according to the education level of participants. In our sample, the final scores ranged from 1 to 10.

\subsubsection{Self-perception}

Self-perception was examined from the perception of future career competence, social competence (or social acceptance), close friendships and global self-worth, four elements in total. This construction respects the classic theory of self-perception (Epstein 1973) and the increasing interest in the "competence" cluster in adolescent studies (Harter 2012; Mruk 1999). Participants responded to the items using a 10-point scale ranging from 1 to 10, with higher scores indicating higher selfperception. EFA indicated that one component could explain 58\% of variance, with support from the scree plot. The validity of the one-factor structure was confirmed by CFA with the robust ML estimation. The model showed a good fit: $\chi^{2}=19.24$, $\mathrm{df}=2, \mathrm{p}<0.001 ; \mathrm{SRMR}=0.02 ; \mathrm{RMSEA}=0.05$ [90\% CI: 0.02-0.09]; CFI $=0.99$. Factor loadings (standardized) were robust, ranging from 0.58 to 0.75 . As to the reliability of this summary scale, the Cronbach alpha coefficient was high at 0.76 .

\subsubsection{Depression}

We selected the depression variable to reflect the mental health aspect of well-being. The scale of depression in CFPS adopted the Center for Epidemiologic Studies Depression Scale (CES-D). This scale is a well-known self-report 20-item scale used to measure depressive symptoms (Radloff 1977). Many studies on social support and well-being (e.g. Ajrouch et al. 2010; Raffaelli et al. 2013; Siedlecki et al. 2014) have adopted CES-D to measure depression due to its high reliability and credibility, including a high 0.88 Cronbach alpha coefficient in one Chinese adolescent sample (Chen et al. 2009). In our sample, the Cronbach alpha coefficient was 0.82 .

\subsubsection{Control variables}

In the current study, socio-demographic characteristics of adolescents served as the control variables, including age, gender, living place, family income and family size. Living in rural and urban areas were coded as 0 and 1 respectively, using the same definition as the Chinese Census Bureau. Variable family income was the net family income of participants in 2011, including wage income, business income, property income, transfer income, and other income, which was categorized into three groups with $33 \%$ and $66 \%$ cut-off points for the analysis. The dichotomy responses of family size - small and large were created by collapsing the original continuous scale. The cutting principle was based on the definition of a nuclear family, so a small family had fewer than 4 family members and a household containing at least 4 members was coded as a large family.

2.3 Statistical Analysis 
Descriptive statistics were examined by Chi-square test (categorical variables) and one-way analysis of variance (continuous variables). Effect sizes were examined by Cramer's V and partial Eta squared. Logistic regression was adopted to investigate the association between parental social support and adolescent health status. Multiple linear regression was used to analyze whether parental social support was related to the academic attainment, self-perception and depression of adolescents. In all the analysis, the two domains of parental social support, emotional support and instrumental support, were analyzed separately. Furthermore, these models were adjusted for age, gender, living location, family income and family size.

SPSS statistical software, version 24 , was used to conduct the analysis.

\section{Results}

Descriptive statistics for adolescents' well-being and socio-demographic characteristics are displayed in Table 1 under different parental social support categories. Adolescents who received high emotional support from their parents more commonly lived in urban areas (53\%), achieved better academic performance, experienced higher self-perception and reported less depression. The 13-year-old group (41\%) and girls (54\%) more commonly received high emotional support from their parents than the other age groups and boys. Although the variance between large and small families was significant (Cramer's $\mathrm{V}=0.121, p<0.001$ ), the homogeneous correlation between family size and different levels of emotional support may be attributed to the small proportion of small families in the sample. A similar but weaker pattern of differences was found for instrumental support, as evidenced by there being no significant variance in academic attainment, gender and family size. Significantly more instrumental support was provided by rural parents, regardless of the levels of the support, which may be influenced by the uneven distribution of living locations. Nevertheless, as with the emotional support domain, adolescents experienced higher levels of self-perception and fewer depressive symptoms when they received high level of instrumental support than when they received low or intermediate levels of instrumental parental social support. Regarding variance in age, instrumental support (Cramer's V=0.107) performed slightly better than emotional support (Cramer's V=0.066). The younger the adolescents were, the more practical support they received from their parents. No statistically significant differences were found between good and suboptimal health groups and between participants from different income groups in either support domain.

Table 2 shows that neither emotional nor instrumental support was associated with self-rated health, after adjustment for socio-demographic characteristics. Of the covariates, only living location was associated with self-rated health in Model 2 (OR=0.54; 95\% CI: 0.33-0.88) and Model 4 (OR=0.53; 95\% CI: 0.32-0.86). Gender, age, family income and family size were not associated with self-rated health.

High emotional support was associated with better academic performance, before $(B=0.13 ; t=4.20)$ and after $(B=0.09$; $\mathrm{t}=2.71$ ) controlling for covariates, as shown in Table 3. Instrumental support was not associated with academic performance. Regardless of the support type, both Models 2 and 4 revealed that academic attainment was associated with gender, age, living location, family size and the middle level of family income.

As Table 4 shows, both the intermediate $(\mathrm{B}=0.19 ; \mathrm{t}=6.53)$ and high $(\mathrm{B}=0.38 ; \mathrm{t}=12.88)$ levels of emotional parental support were associated with adolescent self-perception, compared with the low level. A high level of instrumental support was 
associated with high self-perception $(B=0.21 ; \mathrm{t}=7.17)$. These associations remained in the adjusted models, in which girls and those who came from wealthier families more commonly experienced higher self-perception, regardless of the type of support.

The results in table 5 showed that both intermediate $(B=-0.12 ; \mathrm{t}=-3.98)$ and high $(\mathrm{B}=-0.30 ; \mathrm{t}=-9.93)$ levels of emotional support were negatively associated with depression. Adjustments did not affect these associations. Of the covariates, gender $(\mathrm{B}=-0.06 ; \mathrm{t}=-2.18)$ and living location $(\mathrm{B}=-0.09 ; \mathrm{t}=-3.13)$ were associated with lower levels of depression in the adjusted model. Similarly, the high level of instrumental support had a negative association with depression $(B=-0.14 ; t=-4.72)$. This association was slightly attuned by living location $(B=-0.11 ; \mathrm{t}=-3.71)$ in Model 4 . 
Table 1 Characteristics of the participants, China Family Panel Studies, $2012(N=1306)$

\begin{tabular}{|c|c|c|c|c|c|c|c|c|c|c|c|c|}
\hline \multirow[t]{4}{*}{ Characteristics } & \multirow{4}{*}{$\begin{array}{l}N \\
(\%)\end{array}$} & \multicolumn{11}{|c|}{ Parental social support } \\
\hline & & \multicolumn{3}{|c|}{ Emotional support } & \multicolumn{2}{|c|}{ Difference } & \multicolumn{3}{|c|}{ Instrumental support } & \multicolumn{3}{|c|}{ Difference } \\
\hline & & \multirow{2}{*}{$\begin{array}{l}\text { Low } \\
N(\%)\end{array}$} & \multirow{2}{*}{$\begin{array}{l}\text { Intermediate } \\
N(\%)\end{array}$} & \multirow{2}{*}{$\begin{array}{l}\text { High } \\
N(\%)\end{array}$} & \multirow{2}{*}{$\begin{array}{l}p- \\
\text { value }\end{array}$} & \multirow[t]{2}{*}{ Effect size } & \multirow{2}{*}{$\begin{array}{l}\text { Low } \\
N(\%)\end{array}$} & \multirow{2}{*}{$\begin{array}{l}\text { Intermediate } \\
N(\%)\end{array}$} & \multirow{2}{*}{$\begin{array}{l}\text { High } \\
N(\%)\end{array}$} & \multirow[t]{2}{*}{$p$-value } & \multirow{2}{*}{\multicolumn{2}{|c|}{ Effect size }} \\
\hline & & & & & & & & & & & & \\
\hline \multirow[t]{2}{*}{ Health status } & & & & & 0.054 & Cramer's V & & & & 0.084 & Cramer' & \\
\hline & & & & & & $=0.067$ & & & & & $=0.062$ & \\
\hline \multirow[t]{2}{*}{ Good } & 1210 & $433(92)$ & $398(91)$ & $379(95)$ & & & $494(91)$ & $403(94)$ & $313(95)$ & & & \\
\hline & (93) & & & & & & & & & & & \\
\hline Suboptimal & $96(7)$ & $38(8)$ & $39(9)$ & $19(5)$ & & & $50(9)$ & $28(7)$ & $18(5)$ & & & \\
\hline \multirow{2}{*}{$\begin{array}{l}\text { Academic } \\
\text { attainment }\end{array}$} & 1306 & $471(5.88)$ & $437(6.06)$ & $398(6.40)$ & $<0.001$ & Partial Eta & $544(6.01)$ & $431(6.20)$ & $331(6.12)$ & 0.257 & Partial & Eta \\
\hline & $(6.10)$ & & & & & squared & & & & & squared & \\
\hline \multicolumn{2}{|l|}{ N (Mean) } & & & & & $=0.014$ & & & & & $=0.002$ & \\
\hline \multirow{3}{*}{$\begin{array}{l}\text { Self-perception } \\
\text { N (Mean) }\end{array}$} & 1306 & $471(6.91)$ & $437(7.53)$ & $398(8.16)$ & $<0.001$ & Partial Eta & $544(7.28)$ & $431(7.38)$ & $331(8.02)$ & $<0.001$ & Partial & Eta \\
\hline & $(7.50)$ & & & & & squared & & & & & squared $=$ & 041 \\
\hline & & & & & & $=0.113$ & & & & & & \\
\hline \multirow{3}{*}{$\begin{array}{l}\text { Depression } \\
\text { N (Mean) }\end{array}$} & 1306 & $471(1.69)$ & $437(1.60)$ & $398(1.47)$ & $<0.001$ & Partial Eta & $544(1.63)$ & $431(1.60)$ & $331(1.52)$ & $<0.001$ & Partial & Eta \\
\hline & $(1.59)$ & & & & & squared & & & & & squared & \\
\hline & & & & & & $=0.071$ & & & & & $=0.017$ & \\
\hline \multirow[t]{2}{*}{ Gender } & & & & & 0.003 & Cramer's V & & & & 0.672 & Cramer' & \\
\hline & & & & & & $=0.093$ & & & & & $=0.025$ & \\
\hline \multirow[t]{2}{*}{ Female } & 615 & $203(43)$ & $197(45)$ & $215(54)$ & & & $263(48)$ & $196(46)$ & $156(47)$ & & & \\
\hline & (47) & & & & & & & & & & & \\
\hline \multirow[t]{2}{*}{ Male } & 691 & $268(57)$ & $240(55)$ & $183(46)$ & & & $281(52)$ & $235(55)$ & $175(53)$ & & & \\
\hline & (53) & & & & & & & & & & & \\
\hline Age & & & & & 0.022 & Cramer's V & & & & $<0.001$ & Cramer' & \\
\hline
\end{tabular}




$$
=0.066
$$

$\begin{array}{lllll}11 & 389 & 160(34) & 134(31) & 95(24) \\ & (30) & & & \\ 13 & 470 & 156(33) & 153(35) & 161(41) \\ & (36) & & & \\ 15 & 447 & 155(33) & 150(34) & 142(36) \\ & (34) & & & \end{array}$

Living location

$\begin{array}{lllll}\text { Rural } & 792 & 312(66) & 291(67) & 189(48) \\ & (61) & & & \\ \text { Urban } & 514 & 159(34) & 146(33) & 209(53) \\ & (39) & & & \end{array}$

Family income

Low

$$
436
$$$$
436
$$

Middle

High

Family size

Small

$$
\text { (1) }
$$

$\begin{array}{lll}143(26) & 117(27) & 129(39) \\ 187(34) & 158(37) & 125(38) \\ 214(39) & 156(36) & 77(23)\end{array}$
$<0.001$ Cramer's V

$$
=0.178
$$

$352(65) \quad 272(63) \quad 168(51)$
0.244 Cramer's V

$$
=0.046
$$$$
192(35) \quad 159(37) \quad 163(49)
$$

$<0.001 \quad$ Cramer's V

$$
=0.119
$$
0.104 Cramer's V $=0.054$

$\begin{array}{lll}199(37) & 141(33) & 96(29) \\ 181(33) & 135(31) & 119(36) \\ 164(30) & 155(36) & 116(35)\end{array}$

$<0.001$ Cramer's V $=0.121$

0.307 Cramer's V $=0.043$

$\begin{array}{llll}262 & 79(17) & 74(17) & 109(27)\end{array}$


Large

$1044 \quad 392(83) \quad 363(83) \quad 289(73)$

(80)

Table 2 Odds ratios (95\% confidence intervals) for the association between parental social support and suboptimal health, China Family Panel Studies, 2012 ( $N=1306$ )

\begin{tabular}{|c|c|c|c|c|c|c|c|c|}
\hline & \multicolumn{2}{|l|}{ Model1 $^{\mathrm{a}}$} & \multicolumn{2}{|l|}{ Model $2^{\mathrm{b}}$} & \multicolumn{2}{|l|}{ Model3 $^{a}$} & \multicolumn{2}{|l|}{ Model4 $4^{\mathrm{c}}$} \\
\hline & OR $(95 \% \mathrm{CI})$ & $p$-value & OR $(95 \% \mathrm{CI})$ & $p$-value & OR $(95 \% \mathrm{CI})$ & $p$-value & OR $(95 \% \mathrm{CI})$ & $p$-value \\
\hline \multicolumn{9}{|l|}{ Parental social support } \\
\hline \multicolumn{9}{|l|}{ Emotional support } \\
\hline High & $0.57(0.32-1.01)$ & 0.053 & $0.64(0.36-1.14)$ & 0.132 & & & & \\
\hline $\begin{array}{l}\text { Intermediate } \\
\text { (reference=Low) }\end{array}$ & $1.12(0.70-1.78)$ & 0.644 & $1.12(0.70-1.79)$ & 0.642 & & & & \\
\hline \multicolumn{9}{|l|}{ Instrumental support } \\
\hline High & & & & & $0.57(0.33-0.99)$ & 0.047 & $0.60(0.34-1.06)$ & 0.076 \\
\hline $\begin{array}{l}\text { Intermediate } \\
\text { (reference=Low) }\end{array}$ & & & & & $0.69(0.42-1.11)$ & 0.125 & $0.70(0.43-1.13)$ & 0.141 \\
\hline \multicolumn{9}{|l|}{ Gender } \\
\hline $\begin{array}{l}\text { Male } \\
\text { (reference=Female) }\end{array}$ & & & $0.99(0.65-1.52)$ & 0.966 & & & $1.03(0.68-1.58)$ & 0.884 \\
\hline \multicolumn{9}{|l|}{ Age } \\
\hline 13 & & & $0.90(0.54-1.50)$ & 0.678 & & & $0.85(0.51-1.42)$ & 0.536 \\
\hline $\begin{array}{l}15 \\
(\text { reference }=11)\end{array}$ & & & $0.94(0.56-1.57)$ & 0.804 & & & $0.86(0.51-1.45)$ & 0.573 \\
\hline \multicolumn{9}{|l|}{ Living location } \\
\hline $\begin{array}{l}\text { Urban } \\
\text { (reference=Rural) }\end{array}$ & & & $0.54(0.33-0.88)$ & 0.014 & & & $0.53(0.32-0.86)$ & 0.011 \\
\hline \multicolumn{9}{|l|}{ Family income } \\
\hline High & & & $0.94(0.54-1.62)$ & 0.811 & & & $0.95(0.55-1.64)$ & 0.845 \\
\hline
\end{tabular}


(reference=Low)

Family size

\section{Large}

$($ reference $=$ Small $)$

Nagelkerke R square $\quad 0.012$

0.030

0.009

0.030

${ }^{\mathrm{a}}$ Model 1 and Model 3 are unadjusted.

${ }^{b}$ Model 2 includes gender, age, living location, family income, family size and emotional parental support.

${ }^{\mathrm{c}}$ Model 4 includes gender, age, living location, family income, family size and instrumental parental support

Table 3 Multiple regression model for the association between parental social support and academic attainment, China Family Panel Studies, 2012 (N=1306)

\begin{tabular}{|c|c|c|c|c|c|c|c|c|}
\hline & \multicolumn{2}{|l|}{ Model1 $^{\mathrm{a}}$} & \multicolumn{2}{|l|}{ Model2 ${ }^{\mathrm{b}}$} & \multicolumn{2}{|l|}{ Model3 $^{a}$} & \multicolumn{2}{|l|}{ Model4 ${ }^{\mathrm{c}}$} \\
\hline & Beta $(\mathrm{t})$ & $p$-value & Beta $(\mathrm{t})$ & $p$-value & Beta $(\mathrm{t})$ & $p$-value & Beta $(\mathrm{t})$ & $p$-value \\
\hline \multicolumn{9}{|l|}{ Parental social support } \\
\hline \multicolumn{9}{|l|}{ Emotional support } \\
\hline High & $0.13(4.20)$ & $<0.001$ & $0.09(2.71)$ & 0.007 & & & & \\
\hline $\begin{array}{l}\text { Intermediate } \\
\qquad(\text { reference }=\text { Low })\end{array}$ & $0.05(1.48)$ & 0.140 & $0.04(1.35)$ & 0.178 & & & & \\
\hline \multicolumn{9}{|l|}{ Instrumental support } \\
\hline High & & & & & $0.03(0.92)$ & 0.359 & $0.02(0.64)$ & 0.521 \\
\hline Intermediate & & & & & $0.05(1.63)$ & 0.104 & $0.05(1.72)$ & 0.086 \\
\hline \multicolumn{9}{|l|}{ (reference=Low) } \\
\hline Gender $($ Female $=0)$ & & & $-0.11(-3.93)$ & $<0.001$ & & & $-0.12(-4.23)$ & $<0.001$ \\
\hline Age & & & $0.07(2.52)$ & 0.012 & & & $0.07(2.66)$ & 0.008 \\
\hline Living location (Rural=0) & & & $0.10(3.42)$ & 0.001 & & & $0.11(3.74)$ & $<0.001$ \\
\hline \multicolumn{9}{|l|}{ Family income } \\
\hline High & & & $0.06(1.89)$ & 0.059 & & & $0.06(1.95)$ & 0.052 \\
\hline
\end{tabular}




\section{Middle}

(reference $=$ Low)

Family size (Small=0)

Explained variance $\left(\mathrm{R}^{2}\right)$

Change of variance $\left(\mathrm{R}^{2}\right.$ change)

${ }^{\mathrm{a}}$ Model 1 and Model 3 are unadjusted.

${ }^{b}$ Model 2 includes gender, age, living location, family income, family size and emotional parental support.

${ }^{\mathrm{c}}$ Model 4 includes gender, age, living location, family income, family size and instrumental parental support.

Table 4 Multiple regression model for the association of parental social support and self-perception, China Family Panel Studies, 2012 ( $N=1306$ )

\begin{tabular}{|c|c|c|c|c|c|c|c|c|}
\hline & \multicolumn{2}{|l|}{ Model1 ${ }^{\mathrm{a}}$} & \multicolumn{2}{|l|}{ Model $2^{\mathrm{b}}$} & \multicolumn{2}{|l|}{ Model3 $^{\text {a }}$} & \multicolumn{2}{|l|}{ Model4 ${ }^{\mathrm{c}}$} \\
\hline & $\operatorname{Beta}(\mathrm{t})$ & $p$-value & $\operatorname{Beta}(\mathrm{t})$ & $p$-value & $\operatorname{Beta}(\mathrm{t})$ & $p$-value & $\operatorname{Beta}(\mathrm{t})$ & $p$-value \\
\hline \multicolumn{9}{|l|}{ Parental social support } \\
\hline \multicolumn{9}{|l|}{ Emotional support } \\
\hline High & $0.38(12.88)$ & $<0.001$ & $0.37(12.17)$ & $<0.001$ & & & & \\
\hline Intermediate & $0.19(6.53)$ & $<0.001$ & $0.19(6.46)$ & $<0.001$ & & & & \\
\hline \multicolumn{9}{|l|}{ Instrumental support } \\
\hline High & & & & & $0.21(7.17)$ & $<0.001$ & $0.21(7.05)$ & $<0.001$ \\
\hline Intermediate & & & & & $0.03(1.07)$ & 0.285 & $0.03(1.06)$ & 0.289 \\
\hline \multicolumn{9}{|l|}{ (reference=Low) } \\
\hline Gender $($ Female $=0)$ & & & $-0.05(-1.97)$ & 0.049 & & & $-0.08(-2.97)$ & 0.003 \\
\hline Age & & & $0.002(0.07)$ & 0.945 & & & $0.05(1.84)$ & 0.066 \\
\hline Living location (Rural=0) & & & $0.01(0.46)$ & 0.645 & & & $0.03(0.97)$ & 0.334 \\
\hline \multicolumn{9}{|l|}{ Family income } \\
\hline High & & & $0.07(2.15)$ & 0.031 & & & $0.08(2.40)$ & 0.016 \\
\hline Middle & & & $0.04(1.24)$ & 0.214 & & & $0.04(1.35)$ & 0.177 \\
\hline
\end{tabular}


(reference $=$ Low)

Family size (Small=0)

Explained variance $\left(\mathrm{R}^{2}\right)$

$-0.01(-0.45)$

0.655

$-0.04(-1.31)$

0.192

$<0.001$

0.12

$<0.001$

0.04

$<0.001$

0.06

$<0.001$

Change of variance ( $\mathrm{R}^{2}$ change)

0.11

$<0.001$

0.174

0.04

$<0.001$

0.02

0.001

${ }^{\mathrm{b}}$ Model 2 includes gender, age, living location, family income, family size and emotional parental support.

${ }^{\mathrm{c}}$ Model 4 includes gender, age, living location, family income, family size and instrumental parental support.

Table 5 Multiple regression model for the association of parental social support and depression, China Family Panel Studies, 2012 (N=1306)

\begin{tabular}{|c|c|c|c|c|c|c|c|c|}
\hline & \multicolumn{2}{|l|}{ Model1 $^{\mathrm{a}}$} & \multicolumn{2}{|l|}{ Model $2^{b}$} & \multicolumn{2}{|l|}{ Model3 $^{\mathrm{a}}$} & \multicolumn{2}{|l|}{ Model4 } \\
\hline & Beta $(\mathrm{t})$ & $p$-value & $\operatorname{Beta}(\mathrm{t})$ & $p$-value & $\operatorname{Beta}(\mathrm{t})$ & $p$-value & $\operatorname{Beta}(\mathrm{t})$ & $p$-value \\
\hline \multicolumn{9}{|l|}{ Parental social support } \\
\hline \multicolumn{9}{|l|}{ Emotional support } \\
\hline High & $-0.30(-9.93)$ & $<0.001$ & $-0.29(-9.25)$ & $<0.001$ & & & & \\
\hline $\begin{array}{l}\text { Intermediate } \\
\text { (reference=Low) }\end{array}$ & $-0.12(-3.98)$ & $<0.001$ & $-0.12(-4.03)$ & $<0.001$ & & & & \\
\hline \multicolumn{9}{|l|}{ Instrumental support } \\
\hline High & & & & & $-0.14(-4.72)$ & $<0.001$ & $-0.13(-4.25)$ & $<0.001$ \\
\hline $\begin{array}{l}\text { Intermediate } \\
\text { (reference=Low) }\end{array}$ & & & & & $-0.04(-1.43)$ & 0.154 & $-0.04(-1.36)$ & 0.173 \\
\hline Gender $($ female $=0)$ & & & $-0.06(-2.18)$ & 0.029 & & & $-0.04(-1.28)$ & 0.202 \\
\hline Age & & & $-0.004(-0.17)$ & 0.867 & & & $-0.04(-1.30)$ & 0.194 \\
\hline Living location (Rural=0) & & & $-0.09(-3.13)$ & 0.002 & & & $-0.11(-3.71)$ & $<0.001$ \\
\hline \multicolumn{9}{|l|}{ Family income } \\
\hline High & & & $-0.02(-0.78)$ & 0.439 & & & $-0.03(-1.03)$ & 0.304 \\
\hline Middle & & & $-0.05(-1.57)$ & 0.117 & & & $-0.05(-1.72)$ & 0.085 \\
\hline
\end{tabular}

(reference $=$ Low) 
Family size (Small=0)

$0.03(1.15)$

0.252

$0.05(1.87)$

0.062

Explained variance $\left(\mathrm{R}^{2}\right) \quad 0.07$

$<0.001$

0.09

$<0.001$

0.04

$<0.001$

Change of variance $\left(\mathrm{R}^{2} \quad 0.07\right.$

$<0.001$

0.02

0.002

0.02

$<0.001$

0.02

$<0.001$

change)

${ }^{a}$ Model 1 and Model 3 are unadjusted.

${ }^{\mathrm{b}}$ Model 2 includes gender, age, living location, family income, family size and emotional parental support.

${ }^{\mathrm{c}}$ Model 4 includes gender, age, living location, family income, family size and instrumental parental support. 


\section{Discussion}

The present study of 1306 Chinese adolescents showed that parental social support is associated with adolescent wellbeing. The two types of parental social support manifested various associations with the four indicators of well-being. The associations between emotional support and the four components of well-being were not attributed to potential sociodemographic covariates, except for health status. Low instrumental support was associated with lower self-perception and higher level of depression after controlling for the covariates as well. Broadly speaking, the results indicate a broader valuing of emotional support for adolescent well-being in the current Chinese context.

Neither emotional nor instrumental support was associated with health status after adjusting for socio-demographic covariates. Of the covariates, living in an urban area decreased the likelihood of suboptimal health. Despite China becoming the second largest economy in the world, it has experienced dramatic growth in inequality: for example, its Gini coefficient has ranged between 0.47 and 0.49 over the last decade (SCIO 2013; GOV 2017). However, in this study we did not find a relationship between family income and adolescent health status. Previous evidence has demonstrated that support from social network tends to directly encourage healthier behaviors (Uchino 2006; Uchino et al. 2012; Uchino et al. 1996). Concerning parental social support, the demands or regulations for healthy life habits reduce or buffer adolescents' risky health behaviors (Berge et al. 2010; Patrick et al. 2013; Wake et al. 2007). In this sense, scarcity of health knowledge and lower health education among parents may lead to lower health well-being in rural areas (Liu et al. 2007; Silva-Sanigorski et al. 2012).

Surprisingly, no association was found between instrumental support and adolescents' academic attainment, despite the measurement of the support referring to direct involvement in adolescents' schooling. A high level of emotional support revealed a stable association with a higher score on the cognitive test. The unexpected results of instrumental support advance the understanding of parental involvement in different life stages. There is a broad agreement that parents play an essential role in the excellent academic achievement of Chinese children. The high involvement of parents in schooling is related to traditional norms of self-improvement and high expectations regarding education (Chao and Tseng 2002). Our analysis, nevertheless, provides evidence that direct schooling involvement may no longer be the most efficient mode of support offered by parents at the adolescence stage anymore. Rather, emotional support is the potentially crucial type of parental social support. This situation may be caused by the overly high requirements of the schooling involvement, for example helping to finish homework.

The consistent association between self-perception and social support has been captured by researchers for a long time (Bean et al. 2003; Mcmurray et al. 2011; Noble-Carr et al. 2013). With respect to attachment theory, scholars assert that supportive parenting creates security for children and also conveys feedback for children's self-evaluation (Cohen and Wills 1985). Recently, researchers have expanded the sole secure base function to multiple effects, including nurturing desire, focusing on positive opportunities and facilitating practical strategies (Feeney and Collins 2015). Regarding parental social support specifically, the function of all the benefits relies heavily on one's understanding of optimal parenting. The reason for the positive association between parental social support and the self-concept of adolescents stems partly from the goal of building self-esteem in the suggested family relationships in Western countries. By contrast, in a study conducted in 1990s, the goal of (immigrant) Chinese parents was to foster a harmonious child-parent relationship (Chao 1995), rather than improving the well-being of the children. The traditional parenting aim, however, may be changing: in the present study higher levels of emotional support were associated with a higher level of selfperception. These explanations are consistent with the gradual influence of the concept of "good parenting" in industrial 
countries since the Economic Reform, in which traditional Chinese family values have been challenged by Western parenting norms (Fuligni and Zhang 2004; Wang et al. 2007). As these two norms encounter each other more deeply in globalization, the project of integrating Chinese interdependent family values and Western independent developmental goals is becoming more explicit in Chinese families, a process that has already for some time been reflected among Chinese immigrants (Cheah et al. 2013).

Our study also confirmed the buffering function of parental social support on depression, where emotional support seemed stronger than instrumental support in explaining the variance of depressive symptoms. Wills (2014) proposes two paths for the stress-buffering effects: reducing the impact of risk and increasing the impact of protective factors. The latter understanding, to some extent, corresponds to emerging interest in expanding the buffering function (Feeney and Collins 2015). Parents not only help adolescents to decrease negative emotions, but also empower them to improve their wellbeing in a broader manner. By specifically adding the coping strategies to the measurement of instrumental support, further studies may reveal the buffering function explicitly. Additionally, both instrumental and emotional domains of parental social support consistently disclose an optimal outcome of reducing feelings of sadness, compared with the mixed function of peer support (Desjardins and Leadbeater 2011; Stice et al. 2004).

The key strength of this study is in documenting the various associations between different types of parental social support and multiple perspectives of the well-being of adolescents in contemporary Mainland China. The significant association of parental social support with better academic performance, higher self-perception and lower feelings of depression has further confirmed the shifting family environment of Chinese adolescents. Today in China, adolescents live in a context characterized by several tensions, such as those between traditional family relationship norms and European-American adolescent development values, as well as that between the improvements in general welfare levels and increasing inequality: this has provided an unusual opportunity to examine the association between parental social support and adolescent well-being.

Yet the limitations need to be considered. Only a small proportion of the variance of the well-being of adolescents was associated with parental social support. Rather than identifying the most powerful factors associated with the well-being of adolescents, the purpose of this study was to test the hypothesis that both emotional and instrumental types of parental social support are associated with various domains of well-being. Despite controlling for socio-demographic covariates, it is still possible that there is confounding due to unobserved variables, such as parents' education and occupation. Nevertheless, we included family income as it is also highly related to the previous variables (Liu and Xie 2015). In addition, correlational studies cannot establish a causal relation, so caution should be exercised in interpreting a causal chain from our cross-sectional study (Laursen and Collins 2009).

\section{Conclusion}

The current study highlights the importance of parental social support, given its relevance to adolescent well-being in contemporary Chinese society. Adolescents who receive a high level of support from parents are more likely to have higher academic attainment, better self-perception and lower levels of depression. Both the results of emotional and instrumental support confirm the gradually changed norms of parenting in Chinese families, challenging the single culture heritage explanation. Since the 1980s, incremental changes in market mechanisms have not only led to 10 years of dramatic economic growth in China, but also influenced the family norms of Chinese society via deeper involvement in globalization. The family norms of interdependence are gradually being balanced with the value of autonomy in Western 
countries: combining supportive parenting and communication and, equally importantly, parents' monitoring and control. This new trend in family norms, intertwined with socio-economic inequalities and individual variations, is leading to a distinctive way in which adolescents and their families act, adopt and adjust to the current rapid changes.

Despite it not being a main focus of our study, our research has captured the strong influence of one side-effect of economic development: social inequality. The considerable gaps, especially between rural and urban areas, not only have an impact on adolescent well-being, but also, together with other socio-demographic factors, to some extent restrict the varieties of well-being in different social classes. We are alarmed that the strong power of these structural factors may damage the well-being of adolescents, especially that of rural children. Further studies should take a close look at the changes in parental social support with longitudinal data, which can offer more evidence for the efforts in improving adolescent well-being. Our research furthers the understanding of the association between parental social support and adolescent well-being, located in the interplay between the cultural heritage of family bonding, the macro-level social changes and the globalized norms of adolescents.

Practical suggestions from the results can include more family interventions and services to help parents in providing emotional support, as well as instrumental support. Interventions and services for improving parenting skills could positively influence the well-being of adolescents in China. Many challenges, however, have bedeviled parenting programs in practice, especially in developing countries (see a review Mejia et al. 2012). A plausible example of evidencebased parenting interventions is the implementation of the well-established Parent-Child Interaction Therapy (PCIT) in Hong Kong (Leung et al. 2009). The inclusion of such parenting programs should be considered in the further work of family interventions and services.

\section{References}

Adamson, P. (2007). Child poverty in perspective: An overview of child well-being in rich countries. Report card 7. United Nations Children's Fund. https://www.unicef-irc.org/publications/445/. Accessed 19 May 2016.

Ajrouch, K. J., Reisine, S., Lim, S., Sohn, W., \& Ismail, A. (2010). Perceived everyday discrimination and psychological distress: Does social support matter? Ethnicity \& Health, 15(4), 417-434.

Bean, R. A., Bush, K. R., McKenry, P. C., \& Wilson, S. M. (2003). The impact of parental support, behavioral control, and psychological control on the academic achievement and self-esteem of African American and European American adolescents. Journal of Adolescent Research, 18(5), 523-541.

Ben-Arieh, A., Casas, F., Frønes, I., \& Korbin, J. (2014). Multifaceted concept of child well-being. In A. Ben-Arieh, F. Casas, I. Frønes, \& J. Korbin (Eds.), Handbook of child well-being - theories, methods and policies in global perspective (pp. 1-28). New York: Springer.

Berge, J., Wall, M., Loth, K., \& Neumark-Sztauber, D. (2010). Parenting style as a predictor of adolescent weight and weight-related behaviors. Journal of Adolescent Health, 46(4), 331-338.

Bond, L., Butler, H., Thomas, L., Carlin, J., Glover, S., Bowes, G., \& Patton, G. (2007). Social and school connectedness in early secondary school as predictors of late teenage substance use, mental health, and academic outcomes. Journal of Adolescent Health, 40(4), 357.e359-357.e318.

Bradshaw, J., \& Richardson, D. (2009). An index of child well-being in Europe. Child Indicators Research, 2(3), 319351 .

Brown, T. A. (2015). Confirmatory factor analysis for applied research. New York: The Guilford Press.

Casas, F. (2011). Subjective social indicators and child and adolescent well-being. Child Indicators Research, 4(4), 555575.

Chao, R. K. (1995). Chinese and European American cultural models of the self reflected in mothers' childrearing beliefs. Ethos, 23(3), 328-354.

Chao, R. K., \& Tseng, V. (2002). Parenting in Asians. In M. Bornstein (Ed.), Handbook of parenting: Social conditions and applied parenting (Vol. 4, pp. 59-93). London: Lawrence Erlbaum.

Cheah, C. S. L., Leung, C. Y. Y., \& Zhou, N. (2013). Understanding "tiger parenting" through the perceptions of Chinese immigrant mothers: Can Chinese and U.S. parenting coexist? Asian American Journal of Psychology, 4(1), 30. 
Chen, L., Yang, D., \& Ren, Q. (2015). Report on the state of children in China. Chapinhall at the University of Chicago. http://www.chapinhall.org/research/report/report-state-children-china. Accessed 10 February 2017.

Chen, Z., Yang, X., \& Li, X. (2009). Psychometric features of CES-D in Chinese adolescents. Chinese Journal of Clinical Psychology, 17(4).

Cheng, M., \& Berman, S. L. (2012). Globalization and identity development: A Chinese perspective. Identity Around the World, 138, 103-121.

Cheung, H. S., \& Sim, T. N. (2017). Social support from parents and friends for Chinese adolescents in Singapore. Youth \& Society, 49(4), 548-564.

Chu, P. S., Saucier, D. A., \& Hafner, E. (2010). Meta-analysis of the relationships between social support and well-being in children and adolescents. Journal of Social and Clinical Psychology, 29(6), 624-645.

Cohen, S., \& Wills, T. A. (1985). Stress, social support, and the buffering hypothesis. Psychological bulletin, 98(2), 310357.

Cutrona, C. E., \& Russell, D. W. (1987). The provisions of social relationships and adaptation to stress. Advances in Personal Relationships, 1(1), 37-67.

Desjardins, T. L., \& Leadbeater, B. J. (2011). Relational victimization and depressive symptoms in adolescence: Moderating effects of mother, father, and peer emotional support. Journal of Youth and Adolescence, 40(5), 531544.

Epstein, S. (1973). The self-concept revisitied or a theory of a theory. American Psychologist, 28(5), 404-414.

Feeney, B. C., \& Collins, N. L. (2015). A new look at social support: A theoretical perspective on thriving through relationships. Personality and Social Psychology Review, 19(2), 113-147.

Fuligni, A. J., \& Zhang, W. X. (2004). Attitudes toward family obligation among adolescents in contemporary urban and rural China. Child Development, 75(1), 180-192.

Gordon, M. S., \& Cui, M. (2012). The effect of school-specific parenting processes on academic achievement in adolescence and young adulthood. Family Relations, 61(5), 728-741.

GOV. (2017). National bureau of statistics: China's gini coefficient is generally declining. State Council of the People's Republic of China. http://www.gov.cn/xinwen/2017-01/20/content_5161566.htm. Accessed 10 February 2017.

Haber, M. G., Cohen, J. L., Lucas, T., \& Baltes, B. B. (2007). The relationship between self-reported received and perceived social support: A meta-analytic review. American Journal of Community Psychology, 39(1-2), 133144.

Hair, J. F., Black, W. C., Babin, B. J., \& Anderson, R. E. (2010). Multivariate data analysis : A global perspective (7 edition). New Jersey: Pearson Prentice Hall.

Harter, S. (2012). Susan Harter self-report instruments. The University of Denver Portfolio. https://portfolio.du.edu/SusanHarter/page/44210. Accessed 19 May 2016.

ISSS. (2015). 2012 follow-up data. China Family Panel Studies. http://www.isss.edu.cn/cfps/EN/Data/2012/244.html. Accessed 29 June 2016.

Kristjansson, A. L., Sigfusdottir, I. D., James, J. E., Allegrante, J. P., \& Helgason, A. R. (2010). Perceived parental reactions and peer respect as predictors of adolescent cigarette smoking and alcohol use. Addictive Behaviors, 35(3), 256-259.

Kouvonen, A., De Vogli, R., Stafford, M., Shipley, M. J., Marmot, M. G., Cox, T., Vahtera, J., Vaananen, A., Heponiemi, T., Singh-Manoux, A., Kivimaki, M. (2012). Social support and the likelihood of maintaining and improving levels of physical activity: The whitehall ii study. European Journal of Public Health, 22(4), 514-518.

Lakey, B., \& Orehek, E. (2011). Relational regulation theory: A new approach to explain the link between perceived social support and mental health. Psychological Review, 118(3), 482-495.

Laursen, B., \& Collins, W. A. (2009). Parent-child relationships during adolescence. In L. R. M. \& L. Steinberg (Eds.), Handbook of adolescent psychology (Vol. Contextual influences on adolescent development, pp. 3-42). New Jersey: John Wiley \& Sons.

Leung, C., Tsang, S., Heung, K., \& Yiu, I. (2009). Effectiveness of parent-child interaction therapy (PCIT) among Chinese families. Research on Social Work Practice, 19(3), 304-313.

Liu, A., \& Xie, Y. (2015). Influences of monetary and non-monetary family resource on children's development in verbal ablity in China. Research in Social Stratification and Mobility, 40, 59-70.

Liu, F. (2010). Urban youth in China: Modernity, the internet and the self. New York: Routldege.

Liu, M. N., Zhang, Q. J., Lu, M. S., Kwon, C. S., \& Quan, H. (2007). Rural and urban disparity in health services utilization in China. Medical Care, 45(8), 767-774.

Martorano, B., Natali, L., de Neubourg, C., \& Bradshaw, J. (2014). Child well-being in advanced economies in the late 2000s. Social Indicators Research, 118(1), 247-283.

McMurray, I., Connolly, H., Preston - Shoot, M., \& Wigley, V. (2011). Shards of the old looking glass: Restoring the significance of identity in promoting positive outcomes for looked - after children. Child \& Family Social Work, 16(2), 210-218. 
Mejia, A., Calam, R., \& Sanders, M. R. (2012). A review of parenting programs in developing countries: Opportunities and challenges for preventing emotional and behavioral difficulties in children. Clinical Child and Family Psychology Review, 15(2), 163-175.

Morelli, S. A., Lee, I. A., Arnn, M. E., \& Zaki, J. (2015). Emotional and instrumental support provision interact to predict well-being. Emotion, 15(4), 484-493.

Mruk, C. (1999). Self-esteem: Research, theory and practice. London: Free Association Books.

Noble-Carr, D., Barker, J., \& McArthur, M. (2013). Me, myself and I: Identity and meaning in the lives of vulnerable young people. Canberra: Institute of Child Protection Studies.

Park, S. M., Cho, S. I., \& Moon, S. S. (2010). Factors associated with suicidal ideation: Role of emotional and instrumental support. Journal of Psychosomatic Research, 69(4), 389-397.

Parker, G., Tupling, H., \& Brwon, L. B. (1979). A parental bonding instrument. British Journal of Medical Psychology, 52, $1-10$.

Patrick, H., Hennessy, E., McSpadden, K., \& Oh, A. (2013). Parenting styles and practices in children's obesogenic behaviors: Scientific gaps and future research directions. Childhood Obesity, 9 Supplement, S73-86.

Pollard, E. L., \& Lee, P. D. (2003). Child well-being: A systematic review of the literature. Social Indicators Research, 61(1), 59-78.

Radloff, L. (1977). The CES-D scale: A self-report depression scale for research in the general population. Applied Psychological Measurement, 1(3), 385-401.

Raffaelli, M., Andrade, F. C. D., Wiley, A. R., Sanchez - Armass, O., Edwards, L. L., \& Aradillas - Garcia, C. (2013). Stress, social support, and depression: A test of the stress - buffering hypothesis in a Mexican sample. Journal of Research on Adolescence, 23(2), 283-289.

Riley, A. W. (2004). Evidence that school-age children can self-report on their health. Ambulatory Pediatrics, 4(4), 371376.

Rueger, S. Y., Malecki, C. K., \& Demaray, M. K. (2010). Relationship between multiple sources of perceived social support and psychological and academic adjustment in early adolescence: Comparisons across gender. Journal of Youth and Adolescence, 39(1), 47-61.

Ryan, R. M., Huta, V., \& Deci, E. L. (2008). Living well: A self-determination theory perspective on eudaimonia. Journal of Happiness Studies, 9(1), 139-170.

Ryff, C. (1989). Happiness is everything, or is it? Explorations on the meaning of psychological well-being. Journal of Personality and Social Psychology, 57(6), 1069-1081.

Schermelleh-Engel, K., Moosbrugger, H., \& Müller, H. (2003). Evaluating the fit of structural equation models: Tests of significance and descriptive goodness-of-fit measures. Methods of Psychological Research Online, 8(2), 23-74.

SCIO. (2013). 0.47-0.49: National bureau of statistics first released gini coefficients in the last decade. The State Council Information Office of China. http://www.scio.gov.cn/zhzc/2/32764/Document/1421797/1421797.htm. Accessed 10 February 2017

Seligman, M. (2011). Flourish: A visionary new understanding of happiness and well-being. New York: Free Press.

Shakespeare-Finch, J., \& Obst, P. L. (2011). The development of the 2-way social support scale: A measure of giving and receiving emotional and instrumental support. Journal of Personality Assessment, 93(5), 483-490.

Siedlecki, K. L., Salthouse, T. A., Oishi, S., \& Jeswani, S. (2014). The relationship between social support and subjective well-being across age. Social Indicators Research, 117(2), 561-576.

Silva-Sanigorski, A. d., Ashbolt, R., Green, J., Calache, H., Keith, B., Riggs, E., \& Waters, E. (2012). Parental selfefficacy and oral health-related knowledge are associated with parent and child oral health behaviors and selfreported oral health status. Community Dentistry and Oral Epidemiology, 41(4), 345-352.

Smetana, J. G. (2011). Adolescents, families, and social development: How teens construct their worlds. West Sussex: John Wiely \& Sons.

Stansfeld, S. A., Shipley, M. J., Head, J., Fuhrer, R., \& Kivimaki, M. (2013). Work characteristics and personal social support as determinants of subjective well-being. Plos One, 8(11). e81115.

Stice, E., Ragan, J., \& Randall, P. (2004). Prospective relations between social support and depression: Differential direction of effects for parent and peer support? Journal of Abnormal Psychology, 113(1), 155-159.

Uchino, B. N. (2006). Social support and health: A review of physiological processes potentially underlying links to disease outcomes. Journal of Behavioral Medicine, 29(4), 377-387.

Uchino, B. N. (2009). Understanding the links between social support and physical health: A life-span perspective with emphasis on the separability of perceived and received support. Perspectives on Psychological Science, 4(3), 236-255.

Uchino, B. N., Bowen, K., Carlisle, M., \& Birmingham, W. (2012). Psychological pathways linking social support to health outcomes: A visit with the "ghosts" of research past, present, and future. Social Science \& Medicine, 74(7), 949-957.

Uchino, B. N., Cacioppo, J. T., \& KiecoltGlaser, J. K. (1996). The relationship between social support and physiological processes: A review with emphasis on underlying mechanisms and implications for health. Psychological bulletin, 119(3), 488-531. 
UNICEF. (2012). Progress for children: A report card on adolescents (no.10). United Nations Children's Fund. https://www.unicef.org/publications/index_62280.html. Accessed 21 March 2017.

Varni, J. W., Limbers, C. A., \& Burwinkle, T. M. (2007). How young can children reliably and validly self-report their health-related quality of life?: An analysis of 8,591 children across age subgroups with the pedsql ${ }^{\mathrm{TM}} 4.0$ generic core scales. Health and Quality of Life Outcomes, 5(1), 1.

Wake, M., Nicholson, J. M., Hardy, P., \& Smith, K. (2007). Preschooler obesity and parenting styles of mothers and fathers: Australian national population study. Pediatrics, 120(6), e1520-1527.

Wang, Q., Pomerantz, E. M., \& Chen, H. (2007). The role of parents' control in early adolescents' psychological functioning: A longitudinal investigation in the United States and China. Child Development, 78(5), 1592-1610.

Waterman, A. S. (1993). 2 conceptions of happiness - contrasts of personal expressiveness (eudaimonia) and hedonic enjoyment. Journal of Personality and Social Psychology, 64(4), 678-691.

Wilhelm, K., Niven, H., Parker, G., \& Hadzi-pavlovic, D. (2005). The stability of the parental bonding instrument over a 20-year period. Psychological Medicine, 35, 387-393.

Wills, T. A., Carpenter, M., \& Gibbons, F. X. (2014). Parental and peer support: An analysis of their relations to adolescent substance use. In L. M. Scheier \& W. B. Hansen (Eds.), Parenting and teen drug use (pp. 148-165). New York Oxford.

Wills, T. A., Knight, R., Sargent, J. D., Gibbons, F. X., Pagano, I., \& Williams, R. J. (2017). Longitudinal study of ecigarette use and onset of cigarette smoking among high school students in Hawaii. Tobacco Control, 26(1), 3439.

Wills, T. A., Vaccaro, D., \& Mcnamara, G. (1992). The role of life events, family support, and competence in adolescent substance use - a test of vulnerability and protective factors. American Journal of Community Psychology, 20(3), 349-374.

Xie , Y., \& Hu , J. (2014). An introduction to the China Family Panel Studies (CFPS). Chinese Sociological Review, 47(1), 3-29.

Zhu, S., Tse, S., Cheung, S. H., \& Oyserman, D. (2014). Will I get there? Effects of parental support on children's possible selves. British Journal of Educational Technology, 84(3), 435-453. 